\title{
ANALYTIC CONTINUATION OF THE RESOLVENT OF THE LAPLACIAN ON $\mathrm{SL}(3) / \mathrm{SO}(3)$
}

\author{
RAFE MAZZEO AND ANDRÁS VASY
}

\begin{abstract}
In this paper we continue our program of extending the methods of geometric scattering theory to encompass the analysis of the Laplacian on symmetric spaces of rank greater than one and their geometric perturbations. In our previous work 9 we described the resolvent, and specifically the asymptotic behavior of the Green's function, on $\mathrm{SL}(3) / \mathrm{SO}(3)$ using methods from three-particle scattering. Here we extend the technique of complex scaling to symmetric spaces to show that the resolvent continues analytically across the spectrum.
\end{abstract}

\section{INTRODUCTION}

Harmonic analysis on (both global and local) symmetric spaces is an enduring theme in geometric and harmonic analysis, Lie theory and parts of number theory. The problems arising in spectral synthesis on this class of spaces have been approached from many points of view. This paper is another step in our program to study these questions using techniques from modern geometric scattering theory, particularly as applied to the analysis of quantum $N$-body Hamiltonians. In recent work [9] we have developed machinery to give a microlocal treatment of a parametrix construction for the resolvent of the Laplacian on the symmetric space $\mathrm{SL}(3, \mathbb{R}) / \mathrm{SO}(3, \mathbb{R})$. This produces a complete description of the singularities, particularly at infinity, of this resolvent; however, we treated only the off-spectrum behaviour, for reasons of simplicity and space. Quite recently we became aware of the fact that a full treatment of the analytic continuation of this resolvent past the spectrum does not seem to appear in the literature. (Of course, the analytic continuation of related objects, such as the spherical functions, has been known for some time, cf. [3]. We also mention the paper [14, where some aspects of the continuation problem are discussed from the point of view of explicit formulæ and special functions.) We prove here that the resolvent of the Laplacian on $\mathrm{SL}(3, \mathbb{R}) / \mathrm{SO}(3, \mathbb{R})$ does indeed have an analytic continuation. To do this we use a combination of the microlocal techniques from [9] and the method of complex scaling, which is well-known in scattering theory, and for which this symmetric space setting is well adapted.

We identify $M=\mathrm{SL}(3, \mathbb{R}) / \mathrm{SO}(3, \mathbb{R})$ with the set of real positive definite 3 -by-3 matrices of determinant 1 ; this is a five-dimensional real analytic manifold. The Killing form provides a Riemannian metric $g$. The associated Laplacian $\Delta=\Delta_{g}$ gives a self-adjoint unbounded operator on $L^{2}(M, d g)$, with spectrum $\left[\lambda_{0},+\infty\right)$, $\lambda_{0}=\frac{1}{3}$. Let $R(\lambda)=(\Delta-\lambda)^{-1}$ be the resolvent of $\Delta_{g}, \lambda \notin\left[\lambda_{0},+\infty\right)$. Fix a point $o \in M$, which we may as well assume is the image of the identity matrix $I$ in the

Date: August 14, 2002. 
identification above. The stabilizer subgroup $K_{o}$ (in the natural SL $(3, \mathbb{R})$ action on $M)$ is, of course, isomorphic to $\mathrm{SO}(3, \mathbb{R})$. The Green function $G_{o}(\lambda)$ with pole at $o$ and at eigenvalue $\lambda$ is, by definition $R(\lambda) \delta_{o}$. It is standard that $G_{o}$ lies in the space of $K_{o}$-invariant distributions on $M$.

We fix the branch of the square root function $\sqrt{ }$ on $\mathbb{C} \backslash[0,+\infty)$ which has negative imaginary part when $w \in \mathbb{C} \backslash[0,+\infty)$. Let $S$ denote that part of the Riemann surface for $\lambda \mapsto \sqrt{\lambda-\lambda_{0}}$ where we continue from $\lambda-\lambda_{0} \notin[0,+\infty)$ and allow $\arg \left(\lambda-\lambda_{0}\right)$ to change by any amount less than $\pi$. In other words, starting in the region $\operatorname{Im} \sqrt{\lambda-\lambda_{0}}<0$, we continue across either of the rays where $\operatorname{Im} \sqrt{\lambda-\lambda_{0}}=0$ and $\operatorname{Re} \sqrt{\lambda-\lambda_{0}}>0$, respectively $<0$, allowing the argument of $\sqrt{\lambda-\lambda_{0}}$ to change by any amount less than $\pi / 2$ (so that only the positive imaginary axis is not reached).

We now state our main result:

Theorem 1.1. With all notation as above, the Green function $G_{o}(\lambda)$ continues meromorphically to $S$ as a distribution. Similarly, as an operator between appropriate spaces of $K_{o}$-invariant functions, the resolvent $R(\lambda)$ itself has a meromorphic continuation in this region, with all poles of finite rank.

The poles which arise in this continuation of $R(\lambda)$ are called resonances in scattering theory. Our method does not establish that these poles necessarily exist, and we leave open the fundamental question of whether they actually occur. This theorem also implies that the operator

$$
R(\lambda): L_{c}^{2}(M) \longrightarrow H_{\mathrm{loc}}^{2}(M)
$$

has a meromorphic continuation in the same region, since this is true for its Schwartz kernel; however, since we are no longer restricting to $K_{o}$-invariant function spaces, the poles (if they exist) will no longer be of finite rank!

We expect that this theorem and its proof will generalize to symmetric spaces of arbitrary rank without any essentially new ideas, though of course many new difficulties (of a mostly combinatorial nature) will need to be handled. We expect to return to this soon in a subsequent paper. However, it has seemed worthwhile presenting this method in this simple rank-2 setting, where the techniques of [9] are already in place.

As already noted, a key ingredient in our proof of this continuation is the method of complex scaling. This is an extension of dilation analyticity, and was originally developed in the setting of 2-body scattering by Aguilar-Combes [1] and generalized to the many-body setting by Balslev-Combes [2]. We refer to [6] and [12, Volume 4] for an exposition, and to the paper 13] of Sjöstrand and Zworski for a slightly different point of view. The main new contribution here is an adaptation of this method to the symmetric space setting. Roughly speaking, restricted to the space of $K_{o}$-invariant (or equivalently, Weyl group invariant) functions on the flat, the Laplacian is a three-body type Hamiltonian with the walls of Weyl chambers corresponding to the collision planes. This suggests that many-body methods are natural and appropriate for this problem (and more generally, for analysis on symmetric spaces). Our point of view combines a uniform elliptic theory on all of $M$ with a many-body type analysis on the flats.

To give the reader a rough idea how this works, consider the hyperbolic space $\mathbb{H}^{2}=\mathrm{SL}(2, \mathbb{R}) / \mathrm{SO}(2, \mathbb{R})$, which may be identified with the set of two-by-two positive definite matrices $A$ of determinant 1 . In terms of geodesic normal coordinates 
$(r, \omega)$ about $o=I$, the Laplacian is given by

$$
\Delta_{\mathbb{H}^{2}}=D_{r}^{2}-i(\operatorname{coth} r) D_{r}+(\sinh r)^{-2} D_{\omega}^{2} .
$$

Now consider the diffeomorphism $\Phi_{\theta}: A \mapsto A^{w}, w=e^{\theta}$, on $\mathbb{H}^{2}, \theta \in \mathbb{R}$. This corresponds to dilation along the geodesics through $o$, since these have the form $\gamma_{A}: s \mapsto A^{c s}, c>0$. Thus, in geodesic normal coordinates, $\Phi_{\theta}:(r, \omega) \mapsto\left(e^{\theta} r, \omega\right)$. $\Phi_{\theta}$ defines a group of unitary operators on $L^{2}\left(\mathbb{H}^{2}\right)$ via

$$
\left(U_{\theta} f\right)(A)=\left(\operatorname{det} D_{A} \Phi_{\theta}\right)^{1 / 2}\left(\Phi_{\theta}^{*} f\right)(A), J=\operatorname{det} D_{A} \Phi_{\theta}=w \frac{\sinh w r}{\sinh r}, w=e^{\theta} .
$$

Now, for $\theta$ real, consider the scaled Laplacian

$$
\begin{aligned}
\left(\Delta_{\mathbb{H}^{2}}\right)_{\theta} & =U_{\theta} \Delta_{\mathbb{H}^{2}} U_{\theta}^{-1}=J^{1 / 2} \Phi_{\theta}^{*} \Delta_{\mathbb{H}^{2}} \Phi_{-\theta}^{*} J^{-1 / 2} \\
& =J^{1 / 2}\left(w^{-2} D_{r}^{2}-i w^{-1} \operatorname{coth}(w r) D_{r}+(\sinh (w r))^{-2} D_{\omega}^{2}\right) J^{-1 / 2}
\end{aligned}
$$

This is an operator on $\mathbb{H}^{2}$, with coefficients which extend analytically in the strip $|\operatorname{Im} \theta|<\frac{\pi}{2}$. The square root is continued from the standard branch near $w>$ 0 . (The singularity of the coefficients at $r=0$ is only an artifact of the polar coordinate representation.) Note that $\left(\Delta_{\mathbb{H}^{2}}\right)_{\theta}$ and $\left(\Delta_{\mathbb{H}^{2}}\right)_{\theta^{\prime}}$ are unitary equivalent if $\operatorname{Im} \theta=\operatorname{Im} \theta^{\prime}$ because of the group properties of $U_{\theta}$. The scaled operator, $\left(\Delta_{\mathbb{H}^{2}}\right)_{\theta}$, is not elliptic on all of $\mathbb{H}^{2}$ when $0<|\operatorname{Im} \theta|<\frac{\pi}{2}$ because for $r$ large enough, $w^{2} \sinh (w r)^{-2}$ can lie in $\mathbb{R}^{-}$. However, it is elliptic in some uniform neighbourhood of $o$ in $\mathbb{H}^{2}$, and its radial part

$$
\left(\Delta_{\mathbb{H}^{2}}\right)_{\theta, \mathrm{rad}}=J^{1 / 2}\left(w^{-2} D_{r}^{2}-i w^{-1} \operatorname{coth}(w r) D_{r}\right) J^{-1 / 2},
$$

which corresponds to its action on $\mathrm{SO}(2)$-invariant functions, is elliptic on the entire half-line $r>0$. Thus, a parametrix with compact remainder can be constructed for $\left(\Delta_{\mathbb{H}^{2}}\right)_{\theta \text {,rad }}$, and this show that its essential spectrum lies in $\frac{1}{4}+e^{-2 i \operatorname{Im} \theta}[0,+\infty)$. Hence $\left(\left(\Delta_{\mathbb{H}^{2}}\right)_{\theta \text {,rad }}-\lambda\right)^{-1}$ is meromorphic outside this set. In fact, it is well known that there are no poles in this entire strip (although there are an infinite number on $|\operatorname{Im} \theta|=\pi / 2)$.

Combining this with some more standard technical facts, we are in a position to apply the theory of Aguilar-Balslev-Combes to prove that $\left(\left(\Delta_{\mathbb{H}^{2}}\right)_{\mathrm{rad}}-\lambda\right)^{-1}$, and hence $\left(\Delta_{\mathbb{H}^{2}}-\lambda\right)^{-1}$, has an analytic continuation in $\lambda$ across $\left(\frac{1}{4},+\infty\right)$. This is done by noting that for $\mathrm{SO}(2)$-invariant functions $f, g \in L^{2}\left(\mathbb{H}^{2}\right)$ and $\theta \in \mathbb{R}$,

$$
\left\langle f,\left(\left(\Delta_{\mathbb{H}^{2}}\right)_{\mathrm{rad}}-\lambda\right)^{-1} g\right\rangle=\left\langle U_{\bar{\theta}} f,\left(\left(\Delta_{\mathbb{H}^{2}}\right)_{\theta, \mathrm{rad}}-\lambda\right)^{-1} U_{\theta} g\right\rangle
$$

by the unitarity of $U_{\theta}$. Now if $f, g$ lie in a smaller (dense) class of functions such that $U_{\theta} f$ and $U_{\theta} g$ continue analytically from $\theta \in \mathbb{R}$, then the meromorphic continuation in $\lambda$ of the right hand side is obtained by first making $\theta$ complex with imaginary part of the appropriate sign, and then allowing $\lambda$ to cross the continuous spectrum of $\Delta_{\mathbb{H}^{2}}$ without encountering the essential spectrum of $\left(\Delta_{\mathbb{H}^{2}}\right)_{\theta, \mathrm{rad}}$. Hence the left hand side continues meromorphically also. With some additional care, one can even allow $g$ to be the delta distribution at $o$, yielding the meromorphic continuation of the Green's function.

From the definition of $\Phi_{\theta}$, letting $\theta$ be complex corresponds to deforming $r$ into the complex plane. In fact, we are actually deforming $\mathbb{H}^{2}$ in a family of totally real submanifolds in the complexification $\mathrm{SL}(2, \mathbb{C}) / \mathrm{SO}(2, \mathbb{C})$. As a point of interest, for $\theta \notin \mathbb{R}$, the maximal Grauert tube around $\mathbb{H}^{2}$ has radius $\pi / 2$ and so (since $\operatorname{Im}\left(e^{\theta} r\right)$ is unbounded as $r \rightarrow \infty$ ) the deformed contour is never contained in this Grauert tube. The critical angle $\pi / 2$ is best explained as follows. Recall the decomposition 
of the Lie algebra $\mathfrak{s} l(2, \mathbb{R})=\mathfrak{k}+\mathfrak{p}$, where $\mathfrak{k}=\mathfrak{s o}(2, \mathbb{R})$ and $\mathfrak{p}$ is the space of real 2-by2 symmetric matrices with trace 0 . The Lie algebra of the compact dual of $\mathbb{H}^{2}$ sits inside $\mathfrak{g}_{\mathbb{C}}$ as $\mathfrak{k}+i \mathfrak{p}$, and the compact dual itself is $\exp (i \mathfrak{p})=\mathbb{S}^{2}$. The exponential map on $\mathfrak{g}_{\mathbb{C}}$ has conjugate points only in this $i \mathfrak{p}$ direction (and these must occur since $\mathbb{S}^{2}$ is compact). The submanifolds $\Phi_{\theta}\left(\mathbb{H}^{2}\right)$ avoid these conjugate points until $\arg \theta=\pi / 2$. Notice that in fact $\Phi_{ \pm i \pi / 2}^{*} \Delta_{\mathbb{H}^{2}} \Phi_{\mp i \pi / 2}^{*}=\Delta_{\mathbb{S}^{2}}$ (expressed in normal polar coordinates around some point $o$ ). The poles of the analytic continuation of $\left(\Delta_{\mathbb{H}^{2}}-\lambda\right)^{-1}$ at this critical angle can be explained using this point of view.

The case of $M=\mathrm{SL}(3) / \mathrm{SO}(3)$ is similar, except that both the elliptic analysis of $\Delta_{\theta}$ and the analysis of its radial part (which is now an operator on a 2-dimensional flat) become more interesting: the latter (which is the most crucial part) is where three-body scattering appears in the picture.

The plan of this paper is as follows. In $\S 2$ we review the geometry of the compactification of $M$ and the structure of the Laplacian, and sketch (a modification of) the parametrix construction from [9]. The next section defines and establishes the main facts about complex scaling in our setting. We also prove Theorem 1.1 here, assuming the parametrix construction for the scaled resolvent, to which we turn in $\S 4$. The brief $\S 5$ contains a few comments about further directions and questions.

The authors are grateful to Gilles Carron, Lizhen Ji and Richard Melrose for helpful discussions and for encouragement. R. M. is partially supported by NSF grant \#DMS-0204730; A. V. is partially supported by NSF grant \#DMS-0201092 and a Fellowship from the Alfred P. Sloan Foundation. A. V. also thanks Xue Ping Wang for his kind invitation, and the Université de Nantes for its hospitality, during a stay when the work on this paper started.

\section{The Structure of $M$}

In this section we review some of the material from [9] concerning the geometry of one particularly useful compactification of $M$, and the structure of the Laplacian on it. We refer to that paper for more details.

We first define the compactification $\bar{M}$, which is a $\mathcal{C}^{\infty}$ manifold with corners up to codimension two. It has two boundary hypersurfaces, $H_{\sharp}$ and $H^{\sharp}$, which are perhaps easiest to describe in terms of a natural system of local coordinates derived from the matrix representation of elements in $M$. Write $A \in M$ as $A=O \Lambda O^{t}$, with $O \in \mathrm{SO}(3)$ and $\Lambda$ diagonal. If $\mathfrak{a}$ is the set of diagonal matrices of trace 0 , then $\Lambda \in \exp (\mathfrak{a})$. The ordering of the diagonal entries is undetermined, but in the region where no two of them are equal, then we denote them as $0<\lambda_{1}<\lambda_{2}<\lambda_{3}$ (but recall also that $\left.\lambda_{1} \lambda_{2} \lambda_{3}=1\right)$. In this region the ratios

$$
\mu=\frac{\lambda_{1}}{\lambda_{2}}, \quad \nu=\frac{\lambda_{2}}{\lambda_{3}}
$$

are independent functions, and near the submanifold $\exp (\mathfrak{a})$ in $M$ we can complete them to a full coordinate system by adding the above-diagonal entries $c_{12}, c_{13}, c_{23}$ in the skew-symmetric matrix $T=\log O$. Then $H^{\sharp}=\{\mu=0\}$ and $H_{\sharp}=\{\nu=0\}$, and this coordinate system gives the $\mathcal{C}^{\infty}$ structure near the corner $H_{\sharp} \cap H^{\sharp}$. On the other hand, in a neighborhood of the interior of $H_{\sharp}$, for example, we obtain the compactification and its $\mathcal{C}^{\infty}$ structure using the coordinate system

$$
\mu, s=\lambda_{3}^{-3 / 2}, c_{12}, c_{13}, c_{23} \text {. }
$$


We refer to [9], particularly for an explanation of this odd choice of exponent in the second coordinate, which is necessary in order to make the coordinate change between $(\mu, s)$ and $(\mu, \nu)$ (in the overlap with the corner region) smooth.

The Weyl group $W=S_{3}$ acts on the flat $\exp (\mathfrak{a})$ by permuting the diagonal entries in $\Lambda$. For simplicity we often identify the flat with $\mathfrak{a}$, and in this representation $W$ is generated by Euclidean reflections. The fixed point sets of elements of $W$ partition $\mathfrak{a}$ into the Weyl chambers, and the fixed point sets are called the Weyl chamber walls. We can compactify the flat $\mathfrak{a}$ to a hexagon $\overline{\mathfrak{a}}$ by adding the faces at $\mu=0$ and $\nu=0$; in this picture, the corners $\mu=\nu=0$ appear well away from the closures of the Weyl chamber walls, and the sides of the hexagon are permuted by the action of $W$. The boundaries $H_{\sharp}$ and $H^{\sharp}$ are the orbits of these sides under $\mathrm{SO}(3)$, and adjacent sides of the hexagon lie in different boundary hypersurfaces. The boundary hypersurfaces are each equipped with a fibration, with fibers $\mathrm{SL}(2) / \mathrm{SO}(2)=\mathbb{H}^{2}$ (and base space $\mathbb{R P}^{2}$ ). For example, two interior points of $H^{\sharp}$ are in the same fiber if the sum of the eigenspaces of the two larger eigenvalues (whose ratio is, by assumption, bounded in this region) is the same. The fibrations of these boundary hypersurfaces are compatible at the corner. This gives $\bar{M}$ a boundary fibration structure, similar to (but more complicated than) the ones considered in [11, 10, 7].

We let $\rho_{\sharp}$ and $\rho^{\sharp}$ denote boundary defining functions for $H_{\sharp}$ and $H^{\sharp}$. Fixing $o \in M$, we may assume that $\rho_{\sharp}$ and $\rho^{\sharp}$ are $K_{o}$-invariant. The function $\rho=\rho_{\sharp} \rho^{\sharp}$ is a total boundary defining function. It will be important later to have such a function which is real analytic on $M$, and so we fix the explicit total boundary defining function $\rho=\left(\sum_{i \neq j} \frac{\lambda_{i}}{\lambda_{j}}\right)^{-1}$. Note that this is globally well-defined since it is a symmetric function of the $\lambda_{i}$.

There is a Lie algebra of vector fields associated to this boundary fibration structure, namely the edge-to-edge (or simply ee) Lie algebra $\mathcal{V}_{\text {ee }}(\bar{M})$ consisting of all $\mathcal{C}^{\infty}$ vector fields on $\bar{M}$ which are tangent to the fibers of both boundary hypersurfaces, and in particular tangent to $\partial \bar{M}$. Near the corner, for example, these are spanned over $\mathcal{C}^{\infty}(\bar{M})$ by the basis of sections $\mu D_{\mu}, \nu D_{\nu}, \mu D_{c_{12}}, \nu D_{c_{23}}$ and $\mu \nu D_{c_{13}}$. These generate the algebra of ee differential operators $\operatorname{Diff}_{\mathrm{ee}}(\bar{M})$. Now, $\mathcal{V}_{\text {ee }}(\bar{M})$ is the full set of smooth sections of a vector bundle, ${ }^{\text {ee }} T \bar{M}$, over $\bar{M}$; its dual bundle is ${ }^{\text {ee }} T^{*} \bar{M}$. There is a principal symbol map $\sigma_{\mathrm{ee}, m}$ on Diff ${ }_{\mathrm{ee}}^{m}(\bar{M})$ with range in the space of homogeneous polynomials of degree $m$ on ${ }^{\text {ee }} T^{*} \bar{M}$; this is an extension of the usual principal symbol map on $M$ to the compact space $\bar{M}$. A differential operator $P \in \operatorname{Diff}_{\mathrm{ee}}^{m}(\bar{M})$ is elliptic (in the ee calculus) if $\sigma_{\mathrm{ee}, m}(P)$ is invertible outside the zero section. In particular, the principal symbol of $\Delta$ is $|\zeta|_{g}^{2}, \zeta \in{ }^{\text {ee }} T^{*} \bar{M}$, and so $\Delta$ is an elliptic element of $\operatorname{Diff}_{\text {ee }}^{2}(\bar{M})$.

If $\mathcal{F}$ is any function space, we denote by $\mathcal{F}^{K_{o}}$ the subspace of $K_{o}$-invariant elements. The restriction of $\Delta$ to any one of these $K_{o}$-invariant subspaces is given by its radial part, $\Delta_{\mathrm{rad}}$. This operator plays a key role in the study of spherical functions, see [5]. Moreover, the resolvent $(\Delta-\lambda)^{-1}$ commutes with the $K_{o}$, and its restriction to $K_{o}$-invariant functions is naturally identified with $\left(\Delta_{\mathrm{rad}}-\lambda\right)^{-1}=$ $R(\lambda)_{\text {rad }}$ and is both analytically simpler and more amenable to the scaling method. For this reason, we shall deal almost exclusively with the radial Laplacian rather than the full Laplacian from now on.

An explicit general formula for the radial Laplacian can be found in 5, Chapter II, Proposition 3.9]; in our case it can be calculated directly, cf. [9]. In the 
coordinates $\mu$ and $s$, which are valid near the interior of $H_{\sharp}$,

$$
\begin{aligned}
\Delta_{\mathrm{rad}}= & \frac{1}{3}\left(\left(\mu D_{\mu}\right)^{2}-\left(\frac{\mu+\mu^{-1}}{\mu-\mu^{-1}}-\frac{s^{2}\left(\mu-\mu^{-1}\right)}{s^{4}-s^{2}\left(\mu+\mu^{-1}\right)+1}\right) i \mu D_{\mu}\right) \\
& +\frac{1}{4}\left(\left(s D_{s}\right)^{2}-\frac{2\left(s^{4}-1\right)}{s^{4}-s^{2}\left(\mu+\mu^{-1}\right)+1} i s D_{s}\right) ;
\end{aligned}
$$

there are similar expressions near the interior of $H^{\sharp}$ and near the corner $\mu=\nu=$ 0 . The coefficients of this operator are singular along $\mu=0$, which is a Weyl chamber wall. This singularity is in a certain sense genuine; in fact $\Delta_{\text {rad }}$ has polar coordinate type singularities along all of the Weyl chamber walls, and these are unavoidable because the radial Laplacian is really only an operator on the orbifold $K_{o} \backslash M=\mathfrak{a} / S_{3}$. (A simpler example of this phenomenon is that, written as an ordinary differential operator on $\mathbb{R}^{+}$, the radial part of the Laplacian on $\mathbb{H}^{2}$ has a regular singularity at $r=0$.) The expression (2.1) is also interesting because we can see from it, cf. the discussion preceding Proposition 3.5 below, that the analytic continuations of its coefficients are also singular along the imaginary $\mu$ axis away from $\mu=0$. This is why our method does not provide a continuation of the resolvent into a sector with argument larger than $\pi / 2$.

The remainder of this section is devoted to a sketch of the parametrix construction for $\left(\Delta_{\text {rad }}-\lambda\right)^{-1}$ when $\lambda \notin\left[\lambda_{0},+\infty\right)$. This is meant to provide the reader some frame of reference; the many details we omit can all be found in [9]. We shall present this construction from a slightly different, in fact simpler, point of view than the one in [9] in that we restrict attention from the very beginning to the radial Laplacian. While this could have been done in [9] too, it is absolutely necessary to do so when we apply this construction in $\S 4$ to the resolvent of the scaled radial Laplacian, for reasons we explain below. The trade-off is that we are forced to deal with the singularities of the coefficients of $\Delta_{\text {rad }}$ at the Weyl chamber walls.

Any elliptic parametrix construction is ultimately based upon the invertibility of certain local models for the operator in question. Indeed, the familiar interior parametrix construction for elliptic operators uses the inverses of homogeneous constant coefficient operators obtained by freezing coefficients at any point and inverting the principal part, i.e. the part with the highest homogeneity, modulo an error as indicated below. A convenient way to obtain these inverses in this simplest case is by conjugating by the Fourier transform and dividing by the resulting polynomial. For elliptic operators this polynomial does not vanish outside a compact set, and 'division' is understood as multiplication by the product of the reciprocal of the polynomial, or indeed its principal part, and a function identically 1 near infinity, but vanishing on the compact set. The machinery of pseudodifferential operators provides a mechanism to patch these local inverses together. The same idea can be used for various degenerate problems, such as the one we have here. Now one must find inverses for the model operators, not only at interior points but also at points at infinity. The calculus of ee pseudodifferential operators now provides the formal mechanism for patching these inverses together.

There are two steps in this construction. The first one mimics the local interior parametrix construction, but carried out uniformly in a neighbourhood of the diagonal. This results in the 'small calculus' parametrix, $G_{1}(\lambda)$, which has Schwartz kernel supported in a uniform neighbourhood of the diagonal. The operator $Q_{1}(\lambda)$ 
defined by $\left(\Delta_{\text {rad }}-\lambda\right) G_{1}(\lambda)=I-Q_{1}(\lambda)$ is smoothing, in fact it maps $L^{2}(M)^{K_{o}}$ to $H_{\mathrm{ee}}^{m}(M)^{K_{o}}$ for every $m \in \mathbb{R}$, but is not compact. In the second step, we find a correction term, $G_{2}(\lambda)$, for this parametrix such that $I-(\Delta-\lambda)\left(G_{1}(\lambda)+G_{2}(\lambda)\right)=Q_{2}(\lambda)$ is not only smoothing, but maps into functions with uniform decay at infinity, and thus is compact. This requires that we solve away the 'Taylor series' of $Q_{1}(\lambda)$ at infinity. Even to make sense of this requires that $\mathfrak{a}$ be compactified as a manifold with corners, as we have already done. The construction of $G_{1}(\lambda)$, hence of $Q_{1}(\lambda)$, is more robust and is carried out in [9] for the full Laplacian, rather than just its radial part; in fact, as pointed out there, this does not really use the symmetric space structure of $M$, but only its ee boundary fibration structure. In our context we are faced with some minor new difficulties because of the singularities of $\Delta_{\text {rad }}$ along the Weyl chamber walls, but we explain below how to deal with these. On the other hand, the construction of $G_{2}(\lambda)$ is more delicate and uses certain aspects of the symmetric space structure more strongly.

The key observation in implementing this strategy is that in certain regions of the compactified flat $\overline{\mathfrak{a}}, \Delta_{\text {rad }}$ is modelled by the simpler operators $L_{\sharp \text {,rad }}$ and $L_{\text {rad }}^{\sharp}$. Here

$$
L_{\sharp, \mathrm{rad}}=\frac{1}{4}\left(s D_{s}\right)^{2}+i \frac{1}{2}\left(s D_{s}\right)+\frac{1}{3}\left(\Delta_{\mathbb{H}^{2}}\right)_{\mathrm{rad}},
$$

and $L_{\text {rad }}^{\sharp}$ has a very similar expression; of course, $\left(\Delta_{\mathbb{H}^{2}}\right)_{\mathrm{rad}}=D_{\rho}^{2}-i \operatorname{coth} \rho D_{\rho}$ is the radial ( $\mathrm{SO}(2)$-invariant) part of the Laplacian on $\mathbb{H}^{2}$. The somewhat cumbersome notation here is because these operators are the radial parts of operators $L_{\sharp}$ and $L^{\sharp}$, which are obtained by replacing $\left(\Delta_{\mathbb{H}^{2}}\right)_{\mathrm{rad}}$ by $\Delta_{\mathbb{H}^{2}}$, and which are models for the full Laplacian. Note that, for example, $L_{\sharp \text {,rad }}$ is not $S_{3}$-invariant, but if we restrict it to act on functions supported in a neighbourhood $\mathcal{U}$ of the closure of $H_{\sharp}$ which does not intersect the other Weyl chamber walls, then we can regard it as acting on $S_{3}$ invariant functions on $\mathfrak{a}$. Now we can state more carefully that if $\phi_{\sharp} \in \mathcal{C}_{0}^{\infty}(\mathcal{U})$, then $\phi_{\sharp} L_{\sharp \text {,rad }}$ is a good local approximation to $\Delta_{\text {rad }}$ in the sense that

$$
\phi_{\sharp}\left(\Delta_{\mathrm{rad}}-L_{\sharp}, \mathrm{rad}\right) \in \rho_{\sharp} \operatorname{Diff}_{\mathrm{ee}}^{1}(\overline{\mathfrak{a}}) .
$$

The point is that this error is of only first order and has coefficients vanishing to an extra order at $H_{\sharp}$, which taken together means that it is relatively compact with respect to $\Delta_{\text {rad }}$.

We use these model operators in both steps of the construction. For the first step they are used to resolve the singularities of the radial Laplacian along the Weyl chamber walls. For the second, their resolvents are used to solve away the restrictions of the Schwartz kernel of $Q_{1}(\lambda)$ at the faces $H_{\sharp}$ and $H^{\sharp}$ (and at the corner). We invoke here the analysis of our earlier papers [8, 9] concerning the precise structure of the resolvents of product operators, which is relevant because $L_{\sharp}$ and $L^{\sharp}$ and their radial counterparts are of product type. The final error term $Q_{2}(\lambda)$ is compact on $L^{2}(M)^{K_{o}}$ precisely when $\lambda$ is not in the spectrum of $L_{\sharp \text {,rad }}$ and $L_{\mathrm{rad}}^{\sharp}$, or equivalently, $\lambda \notin\left[\lambda_{0},+\infty\right)$.

We now examine each of the two steps more closely.

As we already indicated, the construction of the first parametrix $G_{1}(\lambda)$ is quite general, and could equally well be carried out in the 'small ee pseudodifferential calculus' for the resolvent of the full Laplacian. However, because we are using the radial Laplacian, we must show how to handle the singularities of this operator at 
the Weyl chamber walls. We shall rely heavily on the fact that $\Delta_{\text {rad }}$ is induced by a quotient space construction. More specifically, the space $\mathfrak{a} / S_{3}$ is naturally identified with $K_{o} \backslash M$ (which is the double coset space $K \backslash G / K$ ), and the operator corresponding to $\Delta_{\text {rad }}$ in this identification lifts to the nonsingular operator $\Delta$ on $M$. Therefore, near the fixed point set of $K_{o}$ we shall use local parametrices for $\Delta$ which are invariant by the local isotropy group, and which hence push down to the quotient.

Let us formalize this more carefully. We divide the compactified flat $\overline{\mathfrak{a}}$ into several different regions as follows. First choose an $S_{3}$-invariant ball $B_{T}(0) \subset$ $\mathfrak{a}$, which we identify with a $K_{o}$-invariant neighbourhood around $o$ in $M$. This corresponds to the fixed points with largest isotropy group. Next, fix two simple roots, which we declare to be positive, and denote $\alpha_{\sharp}, \alpha^{\sharp}$. These determine two 'positive' walls $\mathfrak{w}_{\sharp}=\alpha_{\sharp}^{-1}(0) \cap\left(\alpha^{\sharp}\right)^{-1}\left(\mathbb{R}^{+}\right)$and $\mathfrak{w}^{\sharp}=\left(\alpha^{\sharp}\right)^{-1}(0) \cap\left(\alpha_{\sharp}\right)^{-1}\left(\mathbb{R}^{+}\right)$, which together enclose the positive Weyl chamber $\mathfrak{a}^{+}$. Denote by $\mathfrak{a}_{+, \sharp}$ the union of $\mathfrak{a}^{+}$, its reflection across the wall $\mathfrak{w}_{\sharp}$, and the portion of this wall between them, and similarly for $\mathfrak{a}_{+}^{\sharp}$. We also let $\overline{\mathfrak{a}_{+, \sharp}}=\mathfrak{a}_{+, \sharp} \cup H_{\sharp}$, and similarly for $\overline{\mathfrak{a}_{+}^{\sharp}}$. be the closure Thus $\mathcal{U}^{\sharp}=\left(\left(\alpha^{\sharp}\right)^{-1}((-T, T)) \cap \overline{\mathfrak{a}_{+}^{\sharp}}\right) \backslash B_{T / 2}(0)$ is an $S_{2}$-invariant neighborhood of $\mathfrak{w}^{\sharp}$ which is disjoint from 0 and all other walls, and so we can identify $S_{3}$-invariant functions on $\mathcal{U}^{\sharp}$ with $\mathrm{SO}(2)$-invariant functions on a neighborhood of $\overline{(T / 2,+\infty)} \times o^{\prime}$ in $\overline{(T / 2,+\infty)} \times \mathbb{H}^{2}$ (where $o^{\prime}$ corresponds to $I$ in the matrix representation of $\mathbb{H}^{2}$ ). We define the neighbourhood $\mathcal{U}_{\sharp}$ similarly. Finally, let $\mathcal{U}_{+}$be the $S_{3}$-orbit of the the closure of $\mathfrak{a}_{+}$in $\overline{\mathfrak{a}}$ minus the walls $\mathfrak{w}^{\sharp}$ and $\mathfrak{w}_{\sharp}$. This is summarized in Figure 1 .
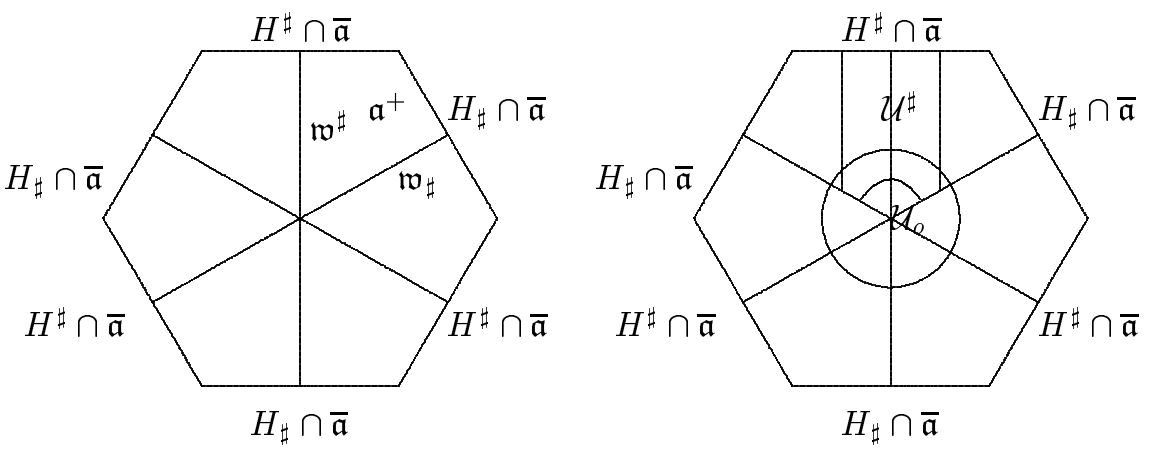

Figure 1. The closure of $\mathfrak{a}$ in the compactifications $\bar{M}$, and the neighborhoods $\mathcal{U}_{o}$ of 0 and $\mathcal{U}^{\sharp}$ of the wall $\mathfrak{w}^{\sharp}$.

We lift each of these neighbourhoods to a different model space, and in each model space we have a different pseudodifferential calculus with which to construct parametrices. Thus $\mathcal{U}_{0}$ lifts to a ball of finite radius in $M$, and we can use ordinary pseudodifferential operators here. $\mathcal{U}^{\sharp}$ and $\mathcal{U}_{\sharp}$ both lift to the model spaces $\mathbb{H}^{2} \times[0,1)$, where we can use the edge calculus, and finally, we identify $\mathcal{U}_{+}$with itself in $\overline{\mathfrak{a}}$ and use the ee calculus here. This now provides the means to treat 'orbifold-elliptic' operators on the quotient $K_{o} \backslash M$.

Definition 2.1. Suppose that $P \in \operatorname{Diffee}_{\mathrm{ee}}^{m}(M)$ is $K_{o}$-invariant. We say that it is radially elliptic if its restriction to each of these neighbourhoods, $\mathcal{U}_{0}, \mathcal{U}^{\sharp}, \mathcal{U}_{\sharp}$ and $\mathcal{U}_{+}$, 
induces operators $P_{0}, P^{\sharp}, P_{\sharp}$ and $P_{+}$, which are symbol-elliptic in the corresponding pseudodifferential calculi on each of the appropriate model spaces.

Let us spell this out in more detail for $\Delta$. It is radial elliptic because:

(i) $\Delta$ is elliptic in a neighbourhood of $o \in M$;

(ii) $\frac{1}{4}\left(s D_{s}\right)^{2}+i \frac{1}{2}\left(s D_{s}\right)+\frac{1}{3} \Delta_{\mathbb{H}^{2}}$ is elliptic in the edge calculus near $[0,1)_{s} \times o^{\prime}$;

(iii) On $\overline{\mathfrak{a}_{+}}$away from the walls,

$$
\Delta_{\mathrm{rad}}=\frac{1}{3}\left(\left(\mu D_{\mu}\right)^{2}+\left(\nu D_{\nu}\right)^{2}-\left(\mu D_{\mu}\right)\left(\nu D_{\nu}\right)+i\left(\mu D_{\mu}\right)+i\left(\nu D_{\nu}\right)\right)+E,
$$

where $E$ is first order combination of the vector fields $\mu D_{\mu}, \nu D_{\nu}$ (and in addition vanishes at the corner), cf. (2.5) in [9], and this operator is elliptic in the ee pseudodifferential calculus (which in this region reduces to the $b$ calculus on a manifold with corners).

Now, let $\left\{\psi_{o}, \psi_{\sharp}, \psi^{\sharp}, \psi_{+}\right\}$be a smooth partition of unity on $\mathfrak{a}$ associated to this covering, such that each function is $S_{3}$-invariant. We also select $S_{3}$-invariant cutoffs $\left\{\chi_{o}, \chi_{\sharp}, \chi^{\sharp}, \chi_{+}\right\}$, such that each element is identically 1 on a neighborhood of the corresponding element in the previous collection. Now suppose that $G_{O}$ is a $K_{O^{-}}$ invariant parametrix for $\Delta-\lambda$ on $B_{T}(0), G_{\sharp}=G^{\sharp}$ is an $\mathrm{SO}(2)$-invariant parametrix for $\frac{1}{4}\left(s D_{s}\right)^{2}+i \frac{1}{2}\left(s D_{s}\right)+\frac{1}{3} \Delta_{\mathbb{H}^{2}}$ on $\mathbb{H}^{2} \times[0,1)_{s}$, and finally, $G_{+}$is a parametrix $\Delta_{\text {rad }}$ on a neighborhood of supp $\psi_{+}$in $\mathfrak{a}_{+}$. We regard each of these operators, suitably localized, as acting on $K_{o}$-invariant functions on $M$. Now set

$$
G_{1}(\lambda)=\chi_{o} G_{o}(\lambda) \psi_{o}+\chi_{\sharp} G_{\sharp}(\lambda) \psi_{\sharp}+\chi^{\sharp} G^{\sharp}(\lambda) \psi^{\sharp}+\chi_{+} G_{+}(\lambda) \psi_{+} .
$$

Then

$$
\left(\Delta_{\mathrm{rad}}-\lambda\right) G_{1}(\lambda)=\operatorname{Id}-Q_{1}(\lambda)
$$

where $Q_{1}(\lambda): L^{2}(M)^{K_{o}} \rightarrow H_{\mathrm{ee}}^{m}(M)^{K_{o}}$ for any $m$.

We now turn to the construction of the correction term. A rather crucial point was omitted in our earlier discussion of this second step in the construction. Namely, while $L_{\sharp \text {,rad }}$ and $L_{\text {rad }}^{\sharp}$ model $\Delta_{\text {rad }}$ on all of $\overline{H_{\sharp}}$, resp. $\overline{H^{\sharp}}$, there is 'no room' to patch their parametrices together on $\overline{\mathfrak{a}}$ for this second step. (Note that this is not an issue in the preceding step because we only used local parametrices for these operators in the interiors of these faces, near the Weyl chamber walls, and the small ee calculus is designed to handle this patching near the corner $H_{\sharp} \cap H^{\sharp}$.) Thus, it turns out that we must perform two additional operations: first, replace the defining functions $\rho_{\sharp}$ and $\rho^{\sharp}$ by $-1 / \log \rho_{\sharp}$ and $-1 / \log \rho^{\sharp}$, respectively, and afterwards blow up the corners of $\overline{\mathfrak{a}}$. The resulting space is denoted $\widetilde{\mathfrak{a}}$, and the closure of $\mathfrak{a}_{+}$inside it is denoted $\widetilde{\mathfrak{a}_{+}}$. The corresponding operations on $M$ produce the space $\widetilde{M}$, and as before, $K_{o} \backslash \widetilde{M}$ is identified with $\widetilde{\mathfrak{a}} / S_{3}$. The closures of the interiors of the faces $H_{\sharp}$ and $H^{\sharp}$ in $\widetilde{M}$ are denoted $\widetilde{H_{\sharp}}$ and $\widetilde{H^{\sharp}}$, respectively. Instead of the neighbourhoods $\mathcal{U}^{\sharp}$ and $\mathcal{U}_{\sharp}$, however, we need larger neighbourhoods: $\tilde{\mathcal{U}}^{\sharp}$ is an $S_{2}$-invariant neighbourhood of $\widetilde{H^{\sharp}} \cap \widetilde{\mathfrak{a}^{+}}$which is disjoint from $\widetilde{H_{\sharp}}$, and similarly for $\tilde{\mathcal{U}}_{\sharp}$. There is no need to consider a neighborhood of 0 , such as $\mathcal{U}_{o}$, since we already have a smoothing error term $Q_{1}(\lambda)$, and we only need to gain decay at infinity to obtain a compact error $Q_{2}(\lambda)$.

Once these operators are lifted to this larger space, we can proceed much as

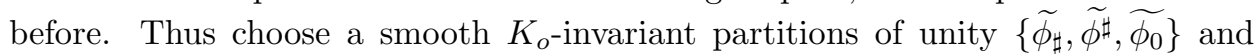
cutoffs $\left\{\widetilde{\psi_{\sharp}}, \widetilde{\psi^{\sharp}}, \widetilde{\psi_{0}}\right\}$, such that the supports of $\widetilde{\phi_{\sharp}}$ and $\widetilde{\psi_{\sharp}}$ are disjoint from $\widetilde{H^{\sharp}}$, and 


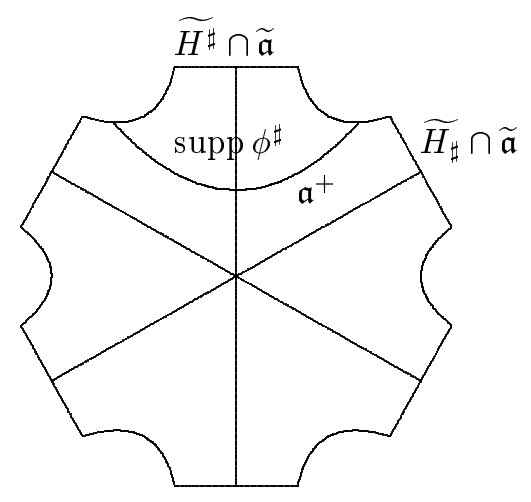

FIGURE 2. $\operatorname{supp} \phi^{\sharp}$ in $\widetilde{\mathfrak{a}}$.

similarly for $\widetilde{\phi^{\sharp}}$ and $\widetilde{\psi^{\sharp}}$, and such that $\widetilde{\psi_{\sharp}}$ is identically one on the support of $\widetilde{\phi_{\sharp}}$, etc. In the supports of each of these we can use the parametrices for the respective model operators, and so we define

$$
\tilde{R}(\lambda)=\widetilde{\psi_{\sharp}} R_{\sharp}(\lambda) \widetilde{\phi_{\sharp}}+\widetilde{\psi^{\sharp}} R^{\sharp}(\lambda) \widetilde{\phi^{\sharp}},
$$

where $R_{\sharp}(\lambda)=\left(L_{\sharp}-\lambda\right)^{-1}, R^{\sharp}(\lambda)=\left(L^{\sharp}-\lambda\right)^{-1}$. It is not difficult to see that

$$
G_{2}(\lambda)=\tilde{R}(\lambda) Q_{1}(\lambda)
$$

has all the desired properties. (We do not need to add a local parametrix for $\Delta-\lambda$ in the support of $\psi_{0}$ since the error $Q_{1}(\lambda)$ is already smoothing.)

Namely, let

$$
G(\lambda)=G_{1}(\lambda)+G_{2}(\lambda), E(\lambda)=(\Delta-\lambda) G(\lambda)-\mathrm{Id}, F(\lambda)=G(\lambda)(\Delta-\lambda)-\mathrm{Id},
$$

and let $x \in \mathcal{C}^{\infty}(M)^{K_{o}}$ be equal to $d(o, .)^{-1}$, the reciprocal of the metric distance function on $M$, outside $B_{1}(o)$. Then for all $m, s, l \in \mathbb{R}$,

$$
\begin{array}{r}
G(\lambda): H_{\mathrm{ee}}^{m}(M)^{K_{o}} \rightarrow H_{\mathrm{ee}}^{m+2}(M)^{K_{o}}, \\
E(\lambda), F(\lambda): H_{\mathrm{ee}}^{m}(M)^{K_{o}} \rightarrow x^{s} H_{\mathrm{ee}}^{l}(M) .
\end{array}
$$

The first line here follows from [9, Corollary 4.8] with $\alpha=0$, and the second part from the proof of [9, Proposition 5.1] (which in turn uses [9, Corollary 4.8]), yielding in fact exponential decay (i.e. $x^{s}$ may be replaced by $e^{-\beta / x}$ for sufficiently small $\beta>0)$. Thus, $E(\lambda), F(\lambda)$ are compact operators on $L^{2}(M)^{K_{o}}$. Hence, analytic Fredholm theory implies that $\left(\Delta_{\text {rad }}-\lambda\right)^{-1}$ is meromorphic on $\mathbb{C} \backslash\left[\frac{1}{3},+\infty\right)$ first as a bounded operator on $L^{2}(M)^{K_{o}}$, and then with the same mapping properties as $G(\lambda)$ by parametrix identities, provided it is invertible for some value of $\lambda$. The self-adjointness and positivity of $\Delta$ in fact show a priori that there are no poles in $\mathbb{C} \backslash[0,+\infty)$.

Later we shall apply this construction, essentially verbatim, to the complex scaled version of the radial Laplacian, $\Delta_{\theta, \text { rad }}$. The reason we have emphasized the use of the radial Laplacian above is that the complex scaling of the full Laplacian, $\Delta_{\text {rad }}$, is not elliptic when $\theta \notin \mathbb{R}$, but fortunately ellipticity is maintained for the radial part. We have already seen this phenomonon in our discussion of the scaling of $\Delta_{\mathbb{H}^{2}}$ 
in the introduction. We note also that the model operators for $\Delta_{\theta \text {,rad }}$ are precisely the scaled versions $L_{\sharp, \theta, \text { rad }}$ and $L_{\theta, \text { rad }}^{\sharp}$ of the radial parts of $L_{\sharp}, L^{\sharp}$, respectively. The construction works just as above so long as $\lambda$ is not in the spectrum of these rescaled model operators.

\section{Complex scaling}

We now introduce the complex scaling for $\Delta$ and $\Delta_{\text {rad }}$. This procedure produces a holomorphic family of operators for which the essential spectrum is shifted. The parametrix construction outlined at the end of the last section is then sufficient to produce a good parametrix, and this leads ultimately to the analytic continuation of the resolvent of $\Delta$.

Let us first review the structure of complex scaling. Fix a connected simply connected domain $D \subset \mathbb{C}$. We wish to define a family of operators $U_{\theta}, \theta \in D$, and dense subspace $\mathcal{A} \subset H_{\mathrm{ee}}^{\infty}(\bar{M})^{K_{o}}$, with the following properties:

(i) If $\theta \in D \cap \mathbb{R}, U_{\theta}$ is unitary on $L^{2}(M)$ and is bounded on all Sobolev spaces, and furthermore, $U_{0}=\mathrm{Id}$;

(ii) For $f \in \mathcal{A}, \theta \rightarrow U_{\theta} f$ has an analytic extension from $D \cap \mathbb{R}$ to all of $D$ with values in $L^{2}(M)^{K_{o}}$;

(iii) The subspace $U_{\theta} \mathcal{A}$ is dense in $L^{2}(M)^{K_{o}}$.

Now, given such a family $U_{\theta}$, we wish to define the family of operators $\Delta_{\theta}=$ $U_{\theta} \Delta U_{\theta}^{-1}$. When $\theta \in \mathbb{R}$, these are defined in the obvious way since $U_{\theta}$ is bounded, and they are all unitarily equivalent. The $\Delta_{\theta}$ are differential operators on $M$ with coefficients that are analytic in $\theta$ in $|\operatorname{Im} \theta|<\frac{\pi}{2}$, as we show below, hence for e.g. $\mathcal{C}_{c}^{\infty}(M), \Delta_{\theta} f$ is analytic in $\theta$. This gives an analytic continuation of $\Delta_{\theta}$ from $\theta \in \mathbb{R}$. The precise statement that we actually need is that $\Delta_{\theta}$ is analytic of type $\mathrm{A}$, see Proposition 3.5.

In complex scaling, one uses the resolvent $\left(\Delta_{\theta}-\lambda\right)^{-1}$ of the scaled operator $\Delta_{\theta}$ to extend $R(\lambda)=(\Delta-\lambda)^{-1}$. More precisely, we show that the matrix elements $\langle f, R(\lambda) g\rangle$ continue to all $\theta \in D$ so long as $f, g \in \mathcal{A}$; using the density of $\mathcal{A}$ this suffices for purposes of spectral theory. In particular, we have $\left(\Delta_{\theta}-\lambda\right)^{-1}=$ $U_{\theta} R(\lambda) U_{\theta}^{-1}$. This equality is valid initially when $\lambda$ is in the resolvent set common to both operators and $\theta$ is real, but after we construct the resolvent $\left(\Delta_{\theta}-\lambda\right)^{-1}$ by parametrix methods, this will give the meromorphic continuation of $R(\lambda)$.

We define a family of unitary operators $U_{\theta}, \theta \in \mathbb{R}$ on $L^{2}(M, d g)$ as follows. First write $w=e^{\theta}>0$ and let $\Phi_{\theta}: M \rightarrow M$ be the diffeomorphism given by $A \mapsto A^{w}$. Then set

$$
\left(U_{\theta} f\right)(A)=\left(\operatorname{det} D_{A} \Phi_{\theta}\right)^{1 / 2} f\left(\Phi_{\theta}(A)\right) .
$$

Hence $U_{\theta}$ is pull-back by $\Phi_{\theta}$ multiplied by the square root of the Jacobian determinant of $\Phi_{\theta}$; this factor is included precisely to make $U_{\theta}$ unitary on $L^{2}(M)$. Clearly $U_{\theta}$ preserves $L^{2}(M)^{K_{o}}$ and is unitary on this subspace.

This definition is motivated as follows. For $A \in M, B=\log A$ is well defined (as the principal branch of the logarithm of a positive definite matrix). The scaling $A \mapsto A^{w}$ corresponds to the more familiar dilation $B \mapsto w B$, which plays an important role in Euclidean scattering.

Note that (not necessarily unit speed) geodesics through $o=I$ are given by $\gamma_{A}: \mathbb{R} \ni s \mapsto A^{c s}, c>0$. Thus, for $\theta \in \mathbb{R}, \Phi_{\theta}$ is dilation along geodesics through $o$. Thus, in geodesic normal coordinates $(r, \omega)$ around $o, \Phi_{\theta}:(r, \omega) \mapsto\left(e^{\theta} r, \omega\right)$. The 
map $A \mapsto A^{w}$ is well defined for all complex $\theta$, and it corresponds to deforming $r$ into a sector of the complex domain. As for the case of $\mathbb{H}^{2}$, we are rotating $M$ into $\mathrm{SL}(3, \mathbb{C}) / \mathrm{SO}(3, \mathbb{C})$, but only to angles which avoid the compact dual $\exp (\mathfrak{k}+i \mathfrak{p})$, cf. the corresponding discussion for $\mathbb{H}^{2}$ in the introduction (also for the language used here).

Although $\Phi_{\theta}$ is a real analytic map on $M$, its regularity properties on $\bar{M}$ are slightly more subtle. The following is easy to verify in local coordinates:

Lemma 3.1. For $\theta \in \mathbb{R}, \Phi_{\theta}$ extends to a homeomorphism of $\bar{M}$; moreover, this extension is a 'conormal diffeomorphism' in the sense that $\Phi_{\theta}^{*}: S^{m}(\bar{M}) \mapsto S^{m w}(\bar{M})$, where $w=e^{\theta}$ as usual and $S^{m}(\bar{M})$ denotes the symbol space. If $C$ denotes a matrix representative of an element of the fiber $\mathbb{H}^{2}=\mathrm{SL}(2) / \mathrm{SO}(2)$ on one of the boundary hypersurfaces, then $\Phi_{\theta}$ is natural in that it induces the map $C \mapsto C^{w}$.

Corollary 3.2. The Jacobian determinant satisfies

$$
\left(\operatorname{det} D_{A} \Phi_{\theta}\right)^{1 / 2} \in \rho^{-w+1} S^{0}(\bar{M})^{K_{o}}, w=e^{\theta},
$$

and it extends analytically to the region

$$
\left\{\theta \in \mathbb{C}:|\operatorname{Im} \theta|<\frac{\pi}{2}\right\} .
$$

Remark 3.3. Another standard way of constructing the family $U_{\theta}$ is via a family of diffeomorphisms generated by some choice of vector field particularly well-adapted to the operator. The work of Christian Gérard [4] contains a good example of this approach. Our choice was dictated by its simplicity and naturality given the matrix structure. In fact, by the general theory of complex scaling, cf. [6. Theorem 16.4], the final analytic continuation of the resolvent does not depend on the choice.

For later purposes we define another family of unitary operators $U_{\theta, T}$, associated to a family of diffeomorphisms $\theta \rightarrow \Phi_{\theta, T}$ of $M$, where each $\Phi_{\theta, T}$ is the identity in the ball $B_{T}(o)$ and equals $\Phi_{\theta}$ outside a compact subset of $M$, and which depends analytically on $\theta$.

To do this, fix $T>0$ and a nondecreasing cutoff function $\phi \in \mathcal{C}^{\infty}(\mathbb{R} ;[0,1])$ which is identically 1 near $\infty$ and identically 0 on $[0, T]$. Now let $r(A)=d(o, A)$, where $d$ is the metric distance, and define

$$
\Phi_{\theta, T}(A)=A^{1+(w-1) \phi(r)}, w=e^{\theta} .
$$

Then $\Phi_{\theta, T}(A)=A$ if $d(o, A) \leq T$, and $\Phi_{\theta, T}(A)=A^{w}$ outside a compact subset of $M$. Moreover, $\Phi_{\theta, T}(A)$ is analytic in $\theta$. We must show that $\Phi_{\theta, T}$ is a diffeomorphism when $\theta$ is real and near 0 , and that it extends analytically to complex $\theta$.

Lemma 3.4. There exists $\delta>0$ such that $\Phi_{\theta, T}: M \rightarrow M$ is a diffeomorphism when $\theta \in \mathbb{R}, e^{\theta}>1-\delta$. In addition, for any $A$, $\left(\operatorname{det} D_{A} \Phi_{\theta, T}\right)^{1 / 2}$ extends analytically to the region

$$
\left\{\theta \in \mathbb{C}:|\operatorname{Im} \theta|<\frac{\pi}{2}, e^{\theta} \notin(-\infty, 1-\delta)\right\} .
$$

Proof. First note that geodesics through $o$ are given by $\left\{A^{s}: s \in \mathbb{R}\right\}$, and $\Phi_{\theta, T}$ preserves these. In fact, in terms of geodesic normal coordinates $(r, \omega) \in[0,+\infty) \times$ $\mathbb{S}^{4}$ centered at $o, \Phi_{\theta, T}$ is given by

$$
(r, \omega) \mapsto((1+(w-1) \phi(r)) r, \omega) .
$$


This is a diffeomorphism provided the derivative of the first component is nonvanishing, i.e.

$$
1+(w-1)\left(\phi(r)+r \phi^{\prime}(r)\right) \neq 0 .
$$

But $\phi(r)+r \phi^{\prime}(r)$ is smooth and equals 1 for $r$ large, hence is bounded; in particular, its range is contained in some interval $\left[0, \delta^{-1}\right]$. Thus $\Phi_{\theta, T}$ is a diffeomorphism provided $-\frac{1}{w-1}$ is not in this range, i.e. if $w \notin(-\infty, 1-\delta]$. This proves the first claim.

The second claim follows immediately from the same calculation. We note that the limitation $|\operatorname{Im} \theta|<\frac{\pi}{2}$ arises because in these coordinates the metric is given by $d r^{2}+h(r, \omega, d \omega)$, and the determinant of $h$ extends to be holomorphic and nonvanishing in this strip.

As before, set

$$
\left(U_{\theta, T} f\right)(A)=\left(\operatorname{det} D_{A} \Phi_{\theta, T}\right)^{1 / 2} f\left(\Phi_{\theta, T}(A)\right) .
$$

The subspace $\mathcal{A}$ is constructed below in Proposition 3.6, and we show there that it has the desired properties for both $U_{\theta}$ and $U_{\theta, T}$.

In any case, because of the geometric nature of this specific choice of $U_{\theta}$, we may define the family of differential operators $\Delta_{\theta}=U_{\theta} \Delta U_{\theta}^{-1}$, without worrying about functional analytic issues of domain. These are all $K_{o}$-invariant on $M$ and have coefficients which depend analytically on $\theta$ in some strip containing the real axis. Examining the expression (2.1), we see that the coefficients there extend holomorphically to $|\operatorname{Im} \theta|<\frac{\pi}{2}$. The delicate term is $\frac{\mu^{-1}+\mu}{\mu^{-1}-\mu} \mu D_{\mu}$. Note that the apparent singularity at $\mu=1$ is only a polar coordinate singularity, as can be seen by setting $r=-\log \mu$, which transforms this term to $-\operatorname{coth} r D_{r}$. However, in the complex scaling, $r$ is replaced by $w r, w=e^{\theta}$, and when $w=i\left(\theta=i \frac{\pi}{2}\right)$, $\operatorname{coth} i r=i \cot r$, which has (non locally integrable) singularities at $r=\pi, 2 \pi, \ldots$. This shows that $|\operatorname{Im} \theta|=\frac{\pi}{2}$ is a genuine boundary for the continuation of this operator.

We have established the smoothness of the coefficients of $\Delta_{\theta}$ when $|\operatorname{Im} \theta|<\frac{\pi}{2}$. However, we also need information about its behaviour at $\partial \bar{M}$, and we turn to this now.

When $\theta \in \mathbb{R}$, the pullbacks of the boundary defining functions $\mu$ and $\nu$ by $\Phi_{\theta}$ are $\mu^{w}$ and $\nu^{w}$, and similarly the total boundary defining function $\rho$ pulls back to $f \rho^{w}$ where $f$ is some smooth nonvanishing function on $\bar{M}$. We then compute the pullbacks of the generating vector fields for the structure algebra $\mathcal{V}_{\mathrm{ee}}$. The vector fields $\mu D_{\mu}$ and $\nu D_{\nu}$ are just multiplied by a factor of $w$ in this pullback, but $\mu D_{c_{12}}, \nu D_{c_{23}}$ and $\mu \nu D_{c_{13}}$ are transformed to to $\mu^{w} D_{c_{12}}, \nu^{w} D_{c_{23}}$ and $\mu^{w} \nu^{w} D_{c_{13}}$, respectively, and these are no longer smooth on $\bar{M}$. This could be remedied by changing the smooth structure, but we do not need to consider this since we are only interested in the radial part of $\Delta_{\theta}$. To see this, suppose we are checking radial ellipticity of the scaled operator near $\mu=1$; then $\mu^{w} D_{c_{12}}$ is smooth here, while $\nu^{w} D_{c_{23}}$ and $\mu^{w} \nu^{w} D_{c_{13}}$ drop out.

Proposition 3.5. If $\theta \in \mathbb{C}$ has $|\operatorname{Im} \theta|<\frac{\pi}{2}$, then $\Delta_{\theta}$ is radially elliptic. The coeffcients of this operator are conormal, but not one-step polyhomogeneous. Moreover, $\theta \rightarrow \Delta_{\theta}$ is an analytic type-A family on $L^{2}(M)^{K_{o}}$ with domain $H_{e e}^{2}(\bar{M})^{K_{o}}$. 
Proof. The first part is easy from the explicit formula. Now, since $\Delta_{\theta}$ is radially elliptic, the domain of $\Delta_{\theta, \mathrm{rad}}$ is $H_{\mathrm{ee}}^{2}(\bar{M})^{K_{o}}$. For any $f \in H_{\mathrm{ee}}^{2}(\bar{M})^{K_{o}}$, the map $\theta \mapsto \Delta_{\theta} f \in L^{2}(M)^{K_{o}}$ is strongly analytic, and this is what it means for $\Delta_{\theta}$ to be an analytic family of type A.

We now state the conclusion of Proposition 4.1 below, that for $|\operatorname{Im} \theta|<\pi / 2$ and as an operator on $L^{2}(M)^{K_{o}}$,

$$
\operatorname{spec}_{\mathrm{ess}}\left(\Delta_{\theta}\right)=\lambda_{0}+e^{-2 i \operatorname{Im} \theta}[0,+\infty) .
$$

In other words, the complex scaling rotates the essential spectrum of $\Delta$ around the threshold at the bottom of the spectrum by an angle $2 \operatorname{Im} \theta$. The proof of this key fact in $\S 4$ uses simple three-body techniques, and is not hard using our geometric approach; we suspect that this is likely to be much less tractable with other, more traditional approaches. Granting this result, we now proceed with the rest of the argument.

We shall write $R(\lambda, \theta)$ for $\left(\Delta_{\theta, \text { rad }}-\lambda\right)^{-1}$. To be definite, in the remainder of this section we shall treat only the analytic continuation of $R(\lambda)_{\mathrm{rad}}=R(\lambda, 0)$ from the lower right quadrant $\operatorname{Im}\left(\lambda-\lambda_{0}\right)<0$ (and through the ray $\left(\lambda_{0},+\infty\right)$ ); the continuation of $R(\lambda)$ from $\operatorname{Im}\left(\lambda-\lambda_{0}\right)$ is handled nearly identically.

Normalize so that $\arg \left(\lambda-\lambda_{0}\right) \in(-\pi, 0)$ for $\operatorname{Im}\left(\lambda-\lambda_{0}\right)<0$. Fix any $\beta \in\left(0, \frac{\pi}{2}\right)$, and define the Riemann surface

$$
S_{\beta}=\left\{\lambda \in S:-\pi<\arg \left(\lambda-\lambda_{0}\right)<2 \beta\right\} .
$$

Thus for $\lambda \in S_{\beta},-\frac{\pi}{2}<\arg \sqrt{\lambda-\lambda_{0}}<\beta$.

The main point, roughly speaking, is that when $-\frac{\pi}{2}<\operatorname{Im} \theta<0, \Delta_{\theta}-\lambda$ is a holomorphic family of operators on $S_{\beta}$ with values in the space of radially elliptic elements in $\operatorname{Diff}_{\mathrm{ee}}^{2}(\bar{M})$. Then, using (3.2), $R(\lambda, \theta)$ is meromorphic in $\lambda$ outside $\lambda_{0}+e^{-2 i \operatorname{Im} \theta}[0,+\infty)$, with values in bounded operators on $L^{2}(M)^{K_{o}}$. This family has only finite rank poles, and these are the poles of the continuation of $R(\lambda)_{\text {rad }}$ if we choose $\theta$ so that $\beta<-\operatorname{Im} \theta<\frac{\pi}{2}$.

More precisely, we shall use the theorem of Aguilar-Balslev-Combes. This theorem has three kinds of hypotheses: some about the scaling $U_{\theta}$, some about the set of analytic vectors $\mathcal{A}$ (these two are summarized in (i)-(iii) at the beginning of $\S 3$ ), and some about the scaled operators $\Delta_{\theta}$. We have explained all of these ingredients except $\mathcal{A}$, and we postpone the discussion of $\mathcal{A}$ until after the statement of the theorem. There, we specify $\mathcal{A}$ directly, as a space of entire functions decaying at infinity, and show that the hypotheses (i)-(iii) at the beginning of $\S 3$ are satisfied for $D=\left\{\theta:|\operatorname{Im} \theta|<\frac{\pi}{4}\right\}$. This guarantees analytic continuation of $R(\lambda)$ to $S_{\pi / 4}$, rather than $S_{\pi / 2}$ as in Theorem 1.1, but the full strength of Theorem 1.1 is obtained subsequently by iterating this argument.

There is a general abstract theorem, due to Nelson, cf. [12, Volume 2], which provides a dense set of analytic vectors for generators of groups of unitary operators, essentially by using the functional calculus, more specifically the spectral projection to bounded subsets of $\mathbb{R}$. Nelson's theorem would allow us to replace $D=\{\theta$ : $\left.|\operatorname{Im} \theta|<\frac{\pi}{4}\right\}$ by $D=\left\{\theta:|\operatorname{Im} \theta|<\frac{\pi}{2}\right\}$ directly in the following theorem, and give the analytic continuation of $R(\lambda)$ to $S_{\pi / 2}$. However, the explicit definition of the subspace $\mathcal{A}$ which we use is intended to make the proof more transparent. 
Theorem. ([6, Theorem 16.4]) Suppose that $U_{\theta}$ and $\mathcal{A}$ satisfy the hypotheses (i)(iii) listed in the beginning of $\S 3$, and that $\Delta_{\theta}$ is a type- $A$ analytic family in the strip $D=\left\{\theta:|\operatorname{Im} \theta|<\frac{\pi}{4}\right\}$, and (3.2) holds for $\theta \in D$. Then

(i) For $f, g \in \mathcal{A},\left\langle f, R(\lambda)_{\mathrm{rad}} g\right\rangle$ has a meromorphic continuation to $S_{\pi / 4}$.

(ii) The poles of the continuation of $\left\langle f, R(\lambda)_{\operatorname{rad}} g\right\rangle$ to $S_{\beta}, \beta<\frac{\pi}{4}$, are the union of all eigenvalues of $\Delta_{\theta, \mathrm{rad}}$ for $\beta<-\operatorname{Im} \theta<\frac{\pi}{4}$.

(iii) The poles are independent of the choice of $U_{\theta}$ in the sense that if $U_{\theta}^{\prime}$ and $\mathcal{A}^{\prime}$ also satisfy (i)-(iii) and if $\mathcal{A} \cap \mathcal{A}^{\prime}$ is dense, then the eigenvalues of $U_{\theta}^{\prime} \Delta_{\mathrm{rad}}\left(U_{\theta}^{\prime}\right)^{-1}$ are the same as those of $\Delta_{\theta, \mathrm{rad}}$.

We briefly outline the proof of the first part since the idea is simple. We must relate $R(\lambda, \theta)$ and $R(\lambda)_{\text {rad }}$, so fix some $\epsilon>0$, and suppose that

$$
\theta \in \Omega_{\epsilon}=\left\{-\epsilon<\operatorname{Im} \theta<\frac{\pi}{4}\right\} \quad \text { and } \quad \arg \left(\lambda-\lambda_{0}\right) \in(-\pi,-\epsilon) .
$$

When $\theta$ is real, $U_{\theta}$ is unitary and so

$$
\left\langle f, R(\lambda)_{\mathrm{rad}} g\right\rangle=\left\langle U_{\bar{\theta}} f,\left(U_{\theta} R(\lambda)_{\mathrm{rad}} U_{\theta}^{-1}\right) U_{\theta} g\right\rangle ;
$$

in addition $U_{\theta} R(\lambda)_{\mathrm{rad}} U_{\theta}^{-1}=R(\lambda, \theta)$, and hence

$$
\left\langle f, R(\lambda)_{\operatorname{rad}} g\right\rangle=\left\langle U_{\bar{\theta}} f, R(\lambda, \theta) U_{\theta} g\right\rangle .
$$

The left side of this equation is independent of $\theta$; on the other hand, the right side is analytic in $\theta$ on $\Omega_{\epsilon}$, and hence is constant on this domain. This holds when $\arg \left(\lambda-\lambda_{0}\right) \in(-\pi,-\epsilon)$.

The extension of $\left\langle f, R(\lambda)_{\operatorname{rad}} g\right\rangle$ to $S_{\beta}$ is done as follows. Take $\theta$ with $-\operatorname{Im} \theta \in$ $\left(\beta, \frac{\pi}{4}\right)$. For $\lambda \in \mathbb{C}$ with $\operatorname{Im}\left(\lambda-\lambda_{0}\right)<0,\left\langle f, R(\lambda)_{\operatorname{rad}} g\right\rangle$ is given by the right hand side of (3.3). But this right side is analytic in $\lambda$ away from the spectrum of $\Delta_{\theta, \mathrm{rad}}$, and meromorphic away from its essential spectrum, hence is meromorphic on $S_{\beta}$, as claimed. This continuation is clearly independent of the choice of $\theta$ with $-\operatorname{Im} \theta \in$ $\left(\beta, \frac{\pi}{4}\right)$ since any such continuation is a meromorphic function of $\lambda$ that agrees with a given function on an open set.

It remains to define the subspace $\mathcal{A}$ of analytic vectors. Let $\mathfrak{a}^{\mathbb{C}}$ denote the complexification of the Cartan subspace $\mathfrak{a}$ (which is identified with the space of diagonal matrices $\exp (\mathfrak{a})$ in $M$ ). The action of the Weyl group $S_{3}$ extends naturally to this complexification. We define $\mathcal{A}$ to be the space of entire $S_{3}$-invariant functions $f$ on $\mathfrak{a}^{\mathbb{C}}$ which decay faster than any power of $\left(1+|z|^{2}\right)^{-1 / 2}$ on every slab $\left\{z \in \mathfrak{a}^{\mathbb{C}}\right.$ : $|\operatorname{Im} z| \leq C|\operatorname{Re} z|\}, 0<C<1$ : for every $0<C<1$ and $N>0$,

$$
\sup _{|\operatorname{Im} z| \leq C|\operatorname{Re} z|}|f(z)|\left(1+|z|^{2}\right)^{N}<+\infty .
$$

Proposition 3.6. When $|\operatorname{Im} \theta|<\frac{\pi}{4}, U_{\theta} \mathcal{A}$ is dense in $L^{2}(M)^{K_{o}}$.

Proof. The space $L^{2}(M)^{K_{o}}$ can be identified with an $L^{2}$ space of $S_{3}$-invariant functions on $\mathfrak{a}$ with respect to a singular measure. In fact, if $d g_{0}$ is the standard Euclidean density on $\mathfrak{a}$ and $\rho$ is a $K_{o}$-invariant total boundary defining function on $\bar{M}$, then there exists a smooth $S_{3}$-invariant function $a$ on $\mathfrak{a}$ with $\rho^{2} a \in \mathcal{C}^{\infty}(\bar{M})^{K_{o}}$ such that $L^{2}(M, d g)^{K_{o}}=L^{2}\left(\mathfrak{a}, a d g_{0}\right)^{S_{3}}$. However, there are effectively polar coordinate singularities which cause $a$ to vanish at the Weyl chamber walls. 
Let $c_{t} e^{-|x|^{2} / t}$ be the fundamental solution of the Euclidean heat equation, $c_{t}=$ $1 / \pi t$. Then if $f \in \mathcal{C}_{c}^{0}(\mathfrak{a})^{S_{3}}$, we let

$$
f_{t}(x)=f * c_{t} e^{-|x|^{2} / t}=\int f(y) c_{t} e^{-|x-y|^{2} / t} d y .
$$

We claim that for $t>0, f_{t} \in \mathcal{A}$. Indeed, if $\theta \in \mathbb{R}, f_{t}\left(e^{\theta} x\right)=\int f(y) c_{t} e^{-\left(e^{\theta} x-y\right)^{2} / t}$, and this continues analytically in $\theta$ because $\exp \left(-(z-y)^{2}\right)$ is entire in $z$ and decreases faster than any power of $\left(1+|z|^{2}\right)^{-1 / 2}$ in $|\operatorname{Im} z|<C|\operatorname{Re} z|$. Finally, since $S_{3}$ acts by reflections and these commute with the heat kernel, each $f_{t}(x)$ is $S_{3}$ invariant. This proves the claim.

To prove that $\mathcal{A}$ is dense in $L^{2}(M, d g)^{K_{o}}$, we note first that $e^{|x|^{2}} f_{t}$ is uniformly bounded when $t<1$, and $\sup e^{|x|^{2}}\left|f(x)-f_{t}(x)\right| \rightarrow 0$ as $t \rightarrow 0$. Since $a e^{-|x|^{2}} \in$ $L^{1}\left(d g_{0}\right)$, we see that for such $f, f_{t} \rightarrow f$ in $L^{2}\left(\mathfrak{a}, a d g_{0}\right)^{S_{3}}$. This is sufficient, since the set of such $f$ is dense in $L^{2}\left(\mathfrak{a}, a d g_{0}\right)^{S_{3}}$.

This argument requires only minor modifications to prove that $U_{\theta} \mathcal{A}$ is also dense in $L^{2}(M, d g)^{K_{o}}$. Namely, for $f$ as above, the function

$$
f_{t}(x)=\int f(y) c_{t} e^{2 \theta} e^{-\left|x-e^{\theta} y\right|^{2} / t} d y \in \mathcal{A},
$$

and we only need to show that $f_{t}\left(e^{\theta} x\right) \rightarrow f$ in $L^{2}\left(\mathfrak{a}, a d g_{0}\right)^{S_{3}}$, or even more simply, that $\sup e^{|x|^{2}}\left|f(x)-f_{t}\left(e^{\theta} x\right)\right| \rightarrow 0$ as $t \rightarrow 0$. However,

$$
f_{t}\left(e^{\theta} x\right)=\int f(y) c_{t} e^{2 \theta} e^{-e^{2 \theta}|x-y|^{2} / t} d y
$$

and $\int c_{t} e^{2 \theta} e^{-e^{2 \theta}|x-y|^{2} / t} d y=1$, and so the standard argument finishes the proof.

Remark 3.7. Note that $e^{-\gamma z^{2}} \rightarrow 0$ as $|z| \rightarrow \infty$ in $|\operatorname{Im} z| \leq(1-\epsilon)|\operatorname{Re} z|$ for any $\epsilon>0$, but this fails when $\epsilon=0$. We have required $\arg e^{\theta}<\frac{\pi}{4}$ to ensure that $U_{\theta}\left(e^{-\gamma x^{2}}\right)$, which is a multiple of $e^{-\gamma\left(e^{\theta} x\right)^{2}}$, lies in $\mathcal{A}$.

Corollary 3.8. For $|\operatorname{Im} \theta|<\frac{\pi}{4}, U_{\theta} \mathcal{A}$ is dense in $H_{e e}^{m}(M)^{K_{o}}$ for all $m>0$.

Proof. Implicit in the definition of these Sobolev spaces, but cf. [9] for an explanation, $(\Delta+1)^{m / 2}: H_{\mathrm{ee}}^{m}(M)^{K_{o}} \rightarrow L^{2}(M)^{K_{o}}$ is an isomorphism. Thus, $f_{t} \rightarrow f$ as $t \rightarrow 0$ in $H_{\mathrm{ee}}^{m}(M)^{K_{o}}$ if and only if $(\Delta+1)^{m / 2} f_{t} \rightarrow(\Delta+1)^{m / 2} f$ in $L^{2}(M)^{K_{o}}$. So given $f \in H_{\mathrm{ee}}^{m}(M)^{K_{o}}$, let $g=(\Delta+1)^{m / 2} f$. Since $\mathcal{A}$ is dense in $L^{2}(M)^{K_{o}}$, there exists a family $g_{t} \in \mathcal{A}$ with $g_{t} \rightarrow g$ as $t \rightarrow 0$ in $L^{2}(M)^{K_{o}}$. Now let $f_{t}=(\Delta+1)^{-m / 2} g_{t}$ and note that $f_{t} \in \mathcal{A}$. Thus, $f_{t} \rightarrow f$ in $H_{\mathrm{ee}}^{m}(M)^{K_{o}}$ as desired.

The continuation of $U_{\theta} g$ may well exist for functions or distributions $g$ which do not lie in $\mathcal{A}$. For example, if $g=\delta_{o}$, the delta distribution at $o$, then its homogeneity shows that for $\theta$ real, $U_{\theta} \delta_{o}=\left(\operatorname{det} D_{o} \Phi_{\theta}\right)^{-1 / 2} \delta_{o}$. Clearly, then, $U_{\theta} \delta_{o}$ extends to be analytic in $\theta$ (e.g. with values in a Sobolev space), and so the Green function, $R(\lambda) \delta_{o}$ also extends via $\left\langle f, R(\lambda) \delta_{o}\right\rangle$ for $f \in \mathcal{A}$.

Note that this does not quite say that $R(\lambda) \delta_{o}$ is a distribution, since that would require that the right hand side of (3.3) be defined for any $f \in \mathcal{C}_{c}^{\infty}(\mathfrak{a})^{S_{3}}$, while for most $f, U_{\bar{\theta}} f$ does not have an analytic extension from the real axis. However, we can prove this using the deformed group of unitary operators, $U_{\theta, T}$, defined in (3.1). Recall that the associated diffeomorphisms $\Phi_{\theta, T}$ fix points in $B_{T}(o)$ and 
equal $\Phi_{\theta}$ for $|x|$ sufficiently large. We use precisely the same arguments as above to establish the density of $U_{\theta, T} \mathcal{A}$. Hence by the uniqueness part of the AguilarBalslev-Combes theorem, the induced analytic extensions agree with one another no matter the value of $T$, and also agree with the extension associated to $U_{\theta}$. But if $f \in \mathcal{C}_{c}^{\infty}\left(B_{T}(o)\right)^{S_{3}}$, then $U_{\theta, T} f=f$ and so $U_{\theta, T} f=f$ has an analytic extension to $\theta \in \mathbb{C}$. Arguing as before, the formula

$$
\left\langle f, R(\lambda)_{\mathrm{rad}} \delta_{o}\right\rangle=\left\langle U_{\bar{\theta}, T} f, R(\lambda, \theta, T) U_{\theta, T} \delta_{o}\right\rangle=\left\langle f, R(\lambda, \theta, T) \delta_{o}\right\rangle
$$

shows that $R(\lambda)_{\mathrm{rad}} \delta_{o}$ does indeed extend analytically as a distribution to $S_{\pi / 2}$ since the right hand side has this property.

Although we have only constructed a subset $\mathcal{A} \subset L^{2}(M)^{K_{o}}$ for which $U_{\theta} \mathcal{A}$ is dense in $L^{2}(M)^{K_{o}}$ when $|\operatorname{Im} \theta|<\pi / 4$, we can still continue $R(\lambda)$ to $S_{\pi / 2}$, for which the formula (3.3) requires larger $\operatorname{Im} \theta$.

Theorem (Theorem 1.1). The Green function $G_{o}(\lambda)$ continues meromorphically to $S_{\pi / 2}$ as a distribution.

Proof. We have shown that the hypotheses of the Aguilar-Balslev-Combes theorem are satisfied for $D=\left\{\theta:|\operatorname{Im} \theta|<\frac{\pi}{4}\right\}$ (for either $U_{\theta}$ or $U_{\theta, T}$ ), except that we still need to show (3.2), a task that we take up in the next section. Hence $R(\lambda)$ continues meromorphically to $S_{\pi / 4}$ in the precise sense of the theorem. In particular, $G_{o}(\lambda)$ continues meromorphically to $S_{\pi / 4}$ as a distribution.

To continue further, we use the following analogue of (3.3):

$$
\left\langle f, R\left(\lambda, \theta^{\prime}\right) g\right\rangle=\left\langle U_{\bar{\theta}} f, R\left(\lambda, \theta+\theta^{\prime}\right) U_{\theta} g\right\rangle
$$

for $f, g \in \mathcal{A},|\operatorname{Im} \theta|<\frac{\pi}{4}$. This is proved similarly to (3.3) using that $U_{\theta} U_{\theta}^{\prime}=U_{\theta+\theta^{\prime}}$, and hence $U_{\theta} R\left(\lambda, \theta^{\prime}\right) U_{\theta}^{-1}=R\left(\lambda, \theta+\theta^{\prime}\right)$. Thus, taking $\theta^{\prime}$ with $\beta<-\operatorname{Im} \theta^{\prime}<\frac{\pi}{4}$, we can continue $R\left(\lambda, \theta^{\prime}\right)$ to $S_{-\operatorname{Im} \theta^{\prime}+\frac{\pi}{4}}$ via (3.5).

This gives the extension of $R(\lambda) \delta_{o}$ to $S_{\pi / 2}$ as a distribution. Indeed, we have shown that such an extension exists in $\mathcal{D}^{\prime}\left(B_{T}(o)\right)$ for any $T>0$. The density of $\mathcal{A}$ implies that these extensions are all the same.

\section{Analysis of the scaled Laplacian}

We take up, at last, the analysis of the scaled Laplacian $\Delta_{\theta}$. The key point here is that for each $\theta$ in the appropriate range, there is a parametrix construction for $\left(\Delta_{\theta}-\lambda\right)^{-1}$ which is almost the same as that for $R(\lambda)$ when $\lambda$ is in the resolvent set. More specifically, the main ingredients in that parametrix construction, as described in $\S 2$, are the radial ellipticity of $\Delta$ and the existence of product-type models at the boundaries of the flat $\exp (\mathfrak{a})$. Both these points have analogues for this scaled operator; indeed, we shall discuss the radial ellipticity below, while the models for $\Delta_{\theta}$ are obtained by conjugating the corresponding models for $\Delta$. For example, the appropriate model for $\Delta_{\theta}$ at $H_{\sharp}$ is the scaling of $L_{\sharp}$,

$$
\left(L_{\sharp}\right)_{\theta}=\frac{1}{4} e^{-2 \theta}\left(s D_{s}\right)^{2}+i \frac{1}{2} e^{-\theta}\left(s D_{s}\right)+\frac{1}{3}\left(\Delta_{\mathbb{H}^{2}}\right)_{\theta},
$$

which equals the conjugate of $L_{\sharp}$ by $U_{\theta}$, and its $\mathrm{SO}(2)$-radial part is $\left(L_{\sharp}\right)_{\theta}$,rad .

The following result is the goal of this parametrix construction.

Proposition 4.1. For $|\operatorname{Im} \theta|<\frac{\pi}{2}$, the essential spectrum of $\Delta_{\theta, \operatorname{rad}}$ on $L^{2}(M)^{K_{o}}$ is given by

$$
\operatorname{spec}_{\mathrm{ess}}\left(\Delta_{\theta, \mathrm{rad}}\right)=\lambda_{0}+e^{-2 i \operatorname{Im} \theta}[0,+\infty)
$$


For technical reasons, it is convenient to work with a unitarily equivalent problem. Let $\rho$ be a $K_{o}$-invariant total boundary defining function. Then multiplication by $\rho^{w}$ gives a unitary map

$$
\rho^{w}: L^{2}\left(M, \rho^{2 \operatorname{Re} w} d g\right)^{K_{o}} \rightarrow L^{2}(M, d g)^{K_{o}},
$$

hence with

$$
\tilde{\Delta}_{\theta, \mathrm{rad}}=\rho^{-w} \Delta_{\theta, \operatorname{rad}} \rho^{w}, w=e^{\theta},
$$

then we have $\left(\Delta_{\theta, \mathrm{rad}}-\lambda\right)^{-1}=\rho^{w}\left(\tilde{\Delta}_{\theta, \mathrm{rad}}-\lambda\right)^{-1} \rho^{-w}$. Therefore it suffices to study the analytic continuation of the resolvent of $\tilde{\Delta}_{\theta \text {, rad }}$ instead. The proposition then follows from the following lemma.

Lemma 4.2. For $|\operatorname{Im} \theta|<\frac{\pi}{2}$, the essential spectrum of $\tilde{\Delta}_{\theta, \operatorname{rad}}$ on $L^{2}(M)^{K_{o}}$ is given by

$$
\operatorname{spec}_{\mathrm{ess}}\left(\tilde{\Delta}_{\theta}\right)=\lambda_{0}+e^{-2 i \operatorname{Im} \theta}[0,+\infty)
$$

Proof. Identify $K_{o}$-invariant functions on $M$ with $S_{3}$-invariant functions on $\mathfrak{a}$, so that $\tilde{\Delta}_{\theta}$ becomes a three-body type perturbation of the scaled Euclidean Laplacian on $\mathfrak{a}$. Using language consonant with that interpretation, the subsystem Hamiltonians are simply the radial part of the scaled Laplacian $\left(\Delta_{\mathbb{H}^{2}}\right)_{\theta}$, conjugated by $\rho_{2}^{w}$ (where $\rho_{2}$ is an $\mathrm{SO}(2)$-invariant boundary defining function on $\mathbb{H}^{2}$ ). The model for $\tilde{\Delta}_{\theta}$ coming from this subsystem is the $\mathrm{SO}(2)$-radial part of

$$
\left(L_{0}\right)_{\theta}=\frac{1}{4} e^{-2 \theta}\left(s D_{s}\right)^{2}+\frac{1}{3} \rho_{2}^{-w}\left(\Delta_{\mathbb{H}^{2}}\right)_{\theta} \rho_{2}^{w}+\frac{1}{4} .
$$

Here $s \in(0,+\infty)$ can be thought of as a variable on the wall of a Weyl chamber. For example, at the wall $\lambda_{1}=\lambda_{2}$ we take $s=\lambda_{3}^{-3 / 2}$. Replacing $s$ by $t=-\log s$ and using the polar variable $r$ on $\mathbb{H}^{2}$, then $\left(L_{0}\right)_{\theta \text {, rad }}$ is a perturbation of $e^{-2 \theta}\left(\frac{1}{4} D_{t}^{2}+\right.$ $\left.\frac{1}{3} D_{r}^{2}\right)+\frac{1}{3}$ as claimed. In fact, using the notation from the end of $\S 2,\left(L_{0}\right)_{\theta}$ is a model operator for $\tilde{\Delta}_{\theta}$ in the sense that for $\phi_{\sharp}$ as in Figure 2,

$$
\phi_{\sharp}\left(\tilde{\Delta}_{\theta}-\left(L_{0}\right)_{\theta}\right) \in \rho_{\sharp}^{w} \operatorname{Diff}_{\mathrm{ee}}^{1}(\bar{M}) .
$$

Note in particular that $\rho_{\sharp}^{w}$ vanishes at the face $\rho_{\sharp}=0$ when $|\operatorname{Im} w|<\frac{\pi}{2}$. Furthermore, as in the introduction, $\operatorname{spec}_{\mathrm{ess}}\left(\rho_{2}^{-w}\left(\Delta_{\mathbb{H}^{2}}\right)_{\theta, \operatorname{rad}} \rho_{2}^{w}\right)=\frac{1}{4}+e^{-2 i \operatorname{Im} \theta}[0,+\infty)$, and hence

$$
\operatorname{spec}_{\text {ess }}\left(L_{0}\right)_{\theta, \operatorname{rad}}=\lambda_{0}+e^{-2 i \operatorname{Im} \theta}[0,+\infty) .
$$

The parametrix construction can now be carried out exactly as at the end of $\S 2$. To do this we use (4.1) and the fact that $\Delta_{\theta}$ remains radially elliptic when $|\operatorname{Im} \theta|<\pi / 2$. This radial ellipticity must be verified on three different regions: near $o \in M$, in a neighbourhood of the Weyl chamber walls, and in the interior of $\mathfrak{a}^{+}$, uniformly out to infinity near the corner in $\overline{\mathfrak{a}}$. In the latter region this is clear since here radial ellipticity simply corresponds to the symbol ellipticity of the scaled Euclidean Laplacian $e^{-2 i \theta} \Delta_{\mathbb{R}^{2}}$ (in the product $b$-calculus). Next, near $\mathfrak{w}_{\sharp}$ say, we need to check that $L_{\sharp, \theta}$ is symbol elliptic in the edge calculus in a neighbourhood of $[0,1)_{s} \times o^{\prime}$ in $[0,+\infty) \times \mathbb{H}^{2}$, and we have checked this already in $\S 1$. Finally, at $o \in M$, the (ordinary!) symbol of $\Delta_{\theta}$ is just $e^{-2 i \theta}|\xi|^{2}$, and hence it too is invertible even in a neighbourhood of $o$. This means that for any allowable $\theta$ we can choose the supports of $\psi_{o}$ etc. small enough so that the first step of the parametrix construction can be carried out. The second stage of the 
parametrix construction can also be done because $\lambda$ avoids the essential spectrum of the model operators. The only step that needs checking is that the analogue of [9, Corollary 4.8] still holds, which in turn only depends on the form of the Schwartz kernel as stated in [8, Theorem 9.3]. This theorem is the consequence of a stationary phase argument, using the form of the Schwartz kernels of the resolvents of $\frac{1}{4} e^{-2 \theta}\left(s D_{s}\right)^{2}$ and $\frac{1}{3} \rho_{2}^{-w}\left(\Delta_{\mathbb{H}^{2}}\right)_{\theta, \mathrm{rad}} \rho_{2}^{w}$. These have the same form as the kernels of the resolvents of $\frac{1}{4}\left(s D_{s}\right)^{2}$ and $\frac{1}{3} \rho_{2}^{-w}\left(\Delta_{\mathbb{H}^{2}}\right)_{\mathrm{rad}} \rho_{2}^{w}$, provided that the shifted spectral parameter $\lambda-\lambda_{0}$ is replaced by $e^{2 \theta}\left(\lambda-\lambda_{0}\right)$. In this way we obtain a parametrix with compact error for $\left(\tilde{\Delta}_{\theta, \text { rad }}-\lambda\right)^{-1}$. This proves that the essential spectrum is $\frac{1}{3}+e^{-2 i \operatorname{Im} \theta}[0,+\infty)$.

This completes the proof of the analytic continuation of the resolvent on $M$.

\section{Future DireCtions}

We expect that this same approach will give the meromorphic continuation of the resolvent of the Laplacian on $\mathrm{SL}(n, \mathbb{R}) / \mathrm{SO}(n, \mathbb{R})$ (and other higher rank symmetric spaces); this continuation will exist on a Riemann surface ramified at points corresonding to poles of all 'subsystem Hamiltonians', i.e. to the poles of the analytic continuations of the resolvents on $\mathrm{SL}(k) / \mathrm{SO}(k), 2 \leq k<n$. In fact, the model operators for $\mathrm{SL}(n) / \mathrm{SO}(n)$ are expected to be sums of operators, each acting on a different factor in a tensor product decomposition, with the operators in each factor being either the radial part of the Laplacian on a lower rank symmetric space or a Euclidean-type operator; this generalizes what we have found here, where the model is a product $\mathbb{R}_{s}^{+} \times \mathrm{SL}(2) / \mathrm{SO}(2)$. This should lead to the following prescription: for a given $n$, take all non-trivial partitions (e.g. for $n=4$, the possibilities are $2+2$ and $3+1$ ); for each partition add the poles of the analytic continuations of $R(\lambda)$ on the corresponding lower rank spaces (possibly shifted by a constant coming from the Euclidean part, as $\left(s D_{s}\right)^{2}$ above). No such branch points occur for $\mathrm{SL}(3) / \mathrm{SO}(3)$ in the region $\arg \left(\lambda-\lambda_{0}\right)<\frac{\pi}{2}$, except possibly at the bottom of the spectrum, since the continuation of $\left(\Delta_{\mathbb{H}^{2}}-\lambda\right)^{-1}$ has no poles up to the same angle.

It is certainly conceivable that the continuation of $R(\lambda)$ on any of the symmetric spaces $\mathrm{SL}(n, \mathbb{R}) / \mathrm{SO}(n, \mathbb{R})$ never has poles (hence no ramification points either), in $\arg \left(\lambda-\lambda_{0}\right)<\frac{\pi}{2}$; it might be possible to prove this using that the residues of these putative poles would correspond to representations of the group which are known not to exist. In any case, this question warrants attention, and this discussion illuminates the importance of determining the existence of poles of the continued resolvent on $\mathrm{SL}(3) / \mathrm{SO}(3)$.

From the point of view of geometric scattering, this continuation sets the stage for many directions of investigation. For example, one should investigate the structure of the scattering operator, the asymptotic distribution of resonances, trace formulae, etc.

\section{REFERENCES}

[1] J. Aguilar and J. M. Combes. A class of analytic perturbations for one-body Schrödinger operators. Comm. Math. Phys., 22 (1971) 269-279.

[2] E. Balslev and J. M. Combes. Spectral properties of many body Schrödinger operators with dilation analytic potentials. Commun. Math. Phys., 22 (1971) 280-294. 
[3] R. Gangolli and V.S. Varadarajan. Harmonic analysis of spherical functions on real reductive groups. Ergebnisse der Mathematik und ihrer Grenzgebiete, Springer-Verlag, Berlin (1988).

[4] C. Gérard. Distortion analyticity for N-particle Hamiltonians. Helv. Phys. Acta, 66 (1993) no. $2,216-225$.

[5] S. Helgason. Groups and geometric analysis. Academic Press, 1984.

[6] P. D. Hislop and I. M. Sigal. Introduction to spectral theory. Springer-Verlag, Berlin (1996).

[7] R. Mazzeo and R. B. Melrose. Pseudodifferential operators on manifolds with fibred boundaries. Asian Journal of Mathematics, 2 (1999) no. 4, 833 - 866.

[8] R. Mazzeo and A. Vasy. Resolvents and Martin boundaries of product spaces. To appear, Geom. Func. Anal. arXiv:math.DG/0012009.

[9] R. Mazzeo and A. Vasy. Scattering theory on $\mathrm{SL}(3) / \mathrm{SO}(3)$ : connections with quantum threebody scattering. Preprint, 2002. arXiv:math.AP/0206118

[10] Rafe Mazzeo. Elliptic theory of differential edge operators. I. Comm. Partial Diff. Eqns., 16 (1991), no. 10, 1615-1664.

[11] R. B. Melrose. Spectral and scattering theory for the Laplacian on asymptotically Euclidian spaces. Marcel Dekker, New York (1994).

[12] M. Reed and B. Simon. Methods of modern mathematical physics. Academic Press, 1979.

[13] Johannes Sjöstrand and Maciej Zworski. Complex scaling and the distribution of scattering poles. J. Amer. Math. Soc., 4(4):729-769, 1991.

[14] N. Wallach. The powers of the resolvent on a locally symmetric space. Bull. Soc. Math. Belg., 42 (1990), 777-795.

R. M.: Department of Mathematics, Stanford University, Stanford, CA 94305

E-mail address: mazzeo@math.stanford.edu

A. V.: Department of Mathematics, Massachusetts Institute of Technology, MA 02139

E-mail address: andras@math.mit.edu 